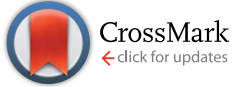

Cite this: RSC Adv., 2017, 7, 17398

Received 10th February 2017

Accepted 15th March 2017

DOI: 10.1039/c7ra01718a

rsc.li/rsc-advances

\section{Enhancing performance of inverted planar perovskite solar cells by argon plasma post- treatment on PEDOT:PSS $\uparrow$}

\author{
Miao Yu,,$^{a}$ Xiaona Huang, $\dot{t}^{\mathrm{a}}$ Shuying Wang, $\dot{t}^{\mathrm{b}}$ Ben Chen, ${ }^{a}$ Yang Zhang, ${ }^{\mathrm{a}}$ Bo Chen, ${ }^{\star c}$ \\ Mingzhen Liu, *a Wanli Zhang ${ }^{a}$ and Jie Xiong*a
}

Interface engineering is an efficient method for improving the performance of inverted planar heterojunction perovskite solar cells (PSCs). In this paper, the PSCs were modified by introducing mild argon plasma post-treatment on poly(3,4-ethylenedioxythiophene):poly(styrene sulfonate) (PEDOT:PSS). The power conversion efficiency (PCE) was enhanced up to $12.17 \%$, compared with $9.55 \%$ of the reference device without interlayer modification. We found that argon plasma treatment on the PEDOT:PSS layer effectively increases its conductivity due to the modification of the PSS ratio in the film. The argon plasma treatment time directly affects the surface morphologies and contact angles of the PEDOT:PSS layer and therefore optimises the uniformity of the PEDOT:PSS layer on the nanometerscale. The improvement in the chemical compositions and film morphologies of PEDOT:PSS could be beneficial to enhancing the wettability and retarding the carrier recombination. The open-circuit voltage $\left(V_{\text {oc }}\right)$ and short circuit current density $\left(J_{S C}\right)$ of PSCs based on the treated PEDOT:PSS were both improved, resulting in the enhancement of PCE.

\section{Introduction}

Organic-inorganic perovskite solar cells have attracted tremendous attention because of their high solar-to-electric power conversion efficiency (PCE) and low fabrication costs. ${ }^{\mathbf{1 - 4}}$ The PCE of perovskite solar cells has been boosted from 3.8\% (ref. 5) to certified $22.1 \%$ (ref. 6) in the past few years. As is known, the device performance is not only affected by the molecular properties of the cell components involved, but also dramatically dependent on the nature of the interfaces present. Hence, interface modification is an efficient method to improve the performance of perovskite solar cells. ${ }^{7}$ For inverted planar heterojunction perovskite solar cells, much effort has been made to modify the interface between the poly(3,4ethylenedioxythiophene):poly(styrene sulfonate) (PEDOT:PSS) anode layer and perovskite active layer. For example, incorporating graphene oxide as a dual functional interface modifier can improve wettability and retard recombination in hybrid

${ }^{a}$ State Key Laboratory of Electronic Thin Films and Integrated Devices, University of Electronic Science and Technology of China, Chengdu 610054, China. E-mail: Jiexiong@uestc.edu.cn; mingzhen.liu@uestc.edu.cn; Fax: +86 2883201232; Tel: +86 2883201231

${ }^{b}$ Chengdu No. 7 High School (GaoxinCampus), Chengdu 610041, China

${ }^{c}$ Institute of Microelectronics of Chinese Academy of Sciences, Beijing 100029, China. E-mail: b_chen@139.com

$\dagger$ Electronic supplementary information (ESI) available. See DOI: 10.1039/c7ra01718a

\$ These authors contribute equally to this work. perovskite solar cells. ${ }^{8}$ Polar solvent $\left(\mathrm{H}_{2} \mathrm{O}\right.$, ethanol, and a mixture) treatment on PEDOT:PSS film was introduced to improve the conductivity but at the cost of a reduction of PCE of perovskite solar cells. ${ }^{9}$ The PCE of CuPc/fullerene-based solar cells could be significantly improved with an appropriate UVozone treatment on PEDOT:PSS. ${ }^{10}$ Recently, it was reported that PEDOT:PSS post-treated with oxygen plasma demonstrated improved conductivity, possibly resulting from a relatively increase of well-conducting PEDOT to less-conducting PSS ratio on the surface. ${ }^{11}$ Meanwhile, they found that the thickness of PEDOT:PSS layers was reduced with an etching rate of $5 \mathrm{~nm}$ $\min ^{-1}$, confirming the removal of materials from the surface of PEDOT:PSS layer. Sometimes the effect of PEDOT:PSS film thickness variations on the device performance has to be considered especially for the pristine layer which has a relatively thin thickness. Atmospheric-pressure argon plasma etched PEDOT:PSS layer was used to improve the performance of organic thin-films solar cells as a hole-transport layer. ${ }^{12,13}$

In this paper, a simple and effective method of argon plasma post-treatment on PEDOT:PSS was proposed to improve the performance of perovskite solar cells. The conductivity, component analysis, film thickness, surface morphology of the plasma-treated PEDOT:PSS films, as well as the wettability of the perovskite precursor solution deposited on these argontreated PEDOT:PSS films were systematically investigated. Subsequently, perovskite solar cells based on argon plasma treated PEDOT:PSS films were fabricated and tested. The PCE of perovskite solar cells increased from $9.55 \%$ for the pristine 
PEDOT:PSS films based device to $9.62 \%, 12.17 \%$ and $10.34 \%$ for the argon plasma treated PEDOT:PSS film based devices by $1 \mathrm{~min}, 3 \mathrm{~min}$ and $5 \mathrm{~min}$, respectively.

\section{Experimental}

\subsection{Experimental materials}

PEDOT:PSS (Clevios ${ }^{\mathrm{TM}}$ PVP. AI 4083) was purchased from Heraeus Materials Technology Shanghai Ltd. $\mathrm{CH}_{3} \mathrm{NH}_{3} \mathrm{I}$ was purchased from Deysol. Lead iodide $\left(\mathrm{PbI}_{2}, 99 \%\right)$, anhydrous $\mathrm{N}, \mathrm{N}$-dimethylformamide (DMF, 99.8\%) and anhydrous chlorobenzene $(\mathrm{CB}, 99.8 \%)$ were purchased from Sigma-Aldrich company. [6,6]-Phenyl- $\mathrm{C}_{61}$-butyric acid methyl ester $\left(\mathrm{PC}_{61} \mathrm{BM}\right.$, $>99 \%$ ) was purchased from Xi'an Polymer Light Technology Corp.

\subsection{PEDOT:PSS films fabrication and characterization}

Firstly, indium tin oxide (ITO) coated on glass $\left(1.5 \times 1.5 \mathrm{~cm}^{2}\right)$ was pre-cleaned by detergent, deionized water, acetone and ethanol in sequence. Then PEDOT:PSS films were deposited on the ITO coated glass by spin-coating the PEDOT:PSS solution at a speed of $5000 \mathrm{rpm}$. After heated at $150{ }^{\circ} \mathrm{C}$ on a hot plate for $10 \mathrm{~min}$, the PEDOT:PSS films were mild argon plasma posttreated by plasma cleaner system with the type of PDC-36G under a base pressure of around $170 \mathrm{~Pa}$ for a certain time of several minutes from 0 to $5 \mathrm{~min}$.

When measuring the conductivity, the PEDOT:PSS films were directly deposited on the glass instead of ITO, and measured by Keithley B2902A system. Silver-indium was welded on the diagonal corners of each PEDOT:PSS film as the electrical contacts for the $J-V$ characteristics measurement. The morphology of the films was observed by atomic force microscopic (AFM, SPI3800, Japan) and scanning electron microscope (SEM, JEOL, JSM-5900 LV). The wettability of $\mathrm{CH}_{3} \mathrm{NH}_{3} \mathrm{PbI}_{3}$ precursor solution $(2.5 \mu \mathrm{L})$ and PEDOT:PSS film surface were measured by drop shape analyzer produced by KRUSS. X-Ray photoelectron spectroscopy (XPS) was carried out with an ESCALAB 250 electron spectrometer from the Chengdu Branch of the Chinese Academy of Sciences.

\subsection{Device fabrication and characterization}

The schematic illustration of the inverted planar heterojunction perovskite solar cells with the structure of ITO/PEDOT:PSS/PVSK/ $\mathrm{PC}_{61} \mathrm{BM} / \mathrm{Ag}$ was shown in Fig. 1. The $\mathrm{CH}_{3} \mathrm{NH}_{3} \mathrm{PbI}_{3}$ perovskite

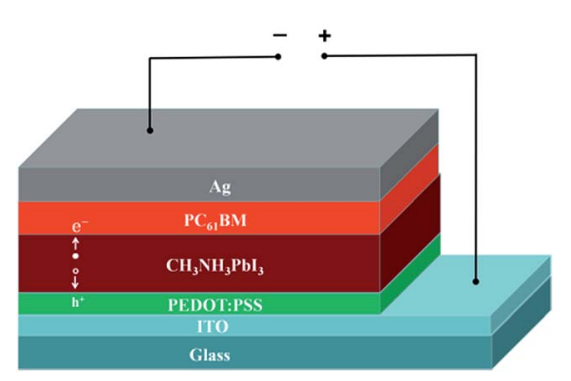

Fig. 1 Schematic illustration of the designed device. active layer was deposited onto the as-prepared PEDOT:PSS layer by spin-coating $\mathrm{CH}_{3} \mathrm{NH}_{3} \mathrm{PbI}_{3}$ precursor solution ( $40 \mathrm{wt} \%$ in DMF) at $4000 \mathrm{rpm}$ for $40 \mathrm{~s}$. In order to improve the crystallization of perovskite active layer deposited by one-step solution method, 70 $\mu \mathrm{L}$ chlorobenzene was dropped quickly onto $\mathrm{CH}_{3} \mathrm{NH}_{3} \mathrm{PbI}_{3}$ wet film about 6 seconds after the beginning of spinning as reported in the literature. ${ }^{14}$ Then the films were annealed at $110{ }^{\circ} \mathrm{C}$ for 30 min inside the glove box filled with nitrogen. After that, a solution of $\mathrm{PC}_{61} \mathrm{BM}$ in chlorobenzene was spin-coated onto the perovskite film at $3000 \mathrm{rpm}$ for $40 \mathrm{~s}$. Finally, a $100 \mathrm{~nm} \mathrm{Ag}$ layer was deposited by thermal evaporation process. The current density-voltage $(J-V)$ measurement was carried out by Keithley model 2400 Source Meter under simulated AM 1.5G solar illumination $\left(100 \mathrm{~mW} \mathrm{~cm}^{-2}\right.$ ) generated by solar simulator (ABET Technologies, SUN 3000).

\section{Results and discussion}

\subsection{Effect of Ar plasma post-treatment on PEDOT:PSS}

Fig. 2 and 3 show top-view SEM and cross-section SEM images of surface morphology of PEDOT:PSS films treated by the argon plasma as a function of treatment time, respectively. A nonuniform distribution of nanometer-scale islands with a diameter of around 50-100 nm was observed on the untreated PEDOT:PSS film. After the argon plasma treatment, the surface morphology of PEDOT:PSS film was observed that the density of islands increased but the size of the islands decreased. The bombardment of argon plasma resulted in a lot of etched small particles about 2-5 $\mathrm{nm}$ on the surface of the islands after $1 \mathrm{~min}$ treatment (Fig. 2b). As the treatment time increased, the density of nanometer-scale particles became higher (Fig. 2c). After 5 min treatment, the particles evolved into the uniformly distributed new islands about 15-20 $\mathrm{nm}$ which have replaced the pristine nonuniform big islands (Fig. 2d).

To investigate the effect induced by plasma post-treatment on the film thickness, a reference thickness was measured by Profilometer Tencor Alfa-step 500. The average thickness reduced from $107 \mathrm{~nm}$ to $84 \mathrm{~nm}$ after $30 \mathrm{~min}$ treatment, suggesting a removal rate of around $0.8 \mathrm{~nm} \mathrm{~min}^{-1}$. It was also

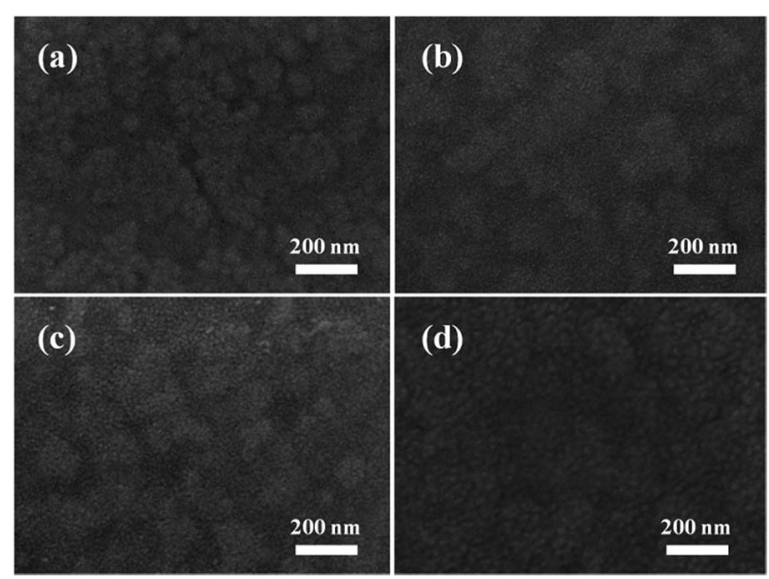

Fig. 2 SEM images of PEDOT:PSS treated by argon plasma for different time: (a) untreated, (b) 1 min, (c) 3 min, (d) 5 min. 

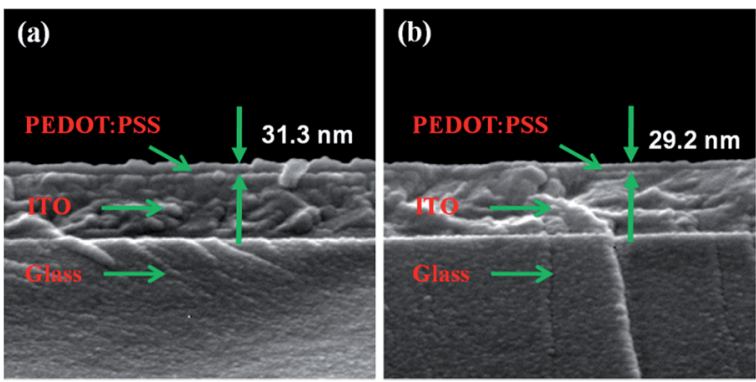

Fig. 3 Comparison of the cross-section SEM images of PEDOT:PSS treated by argon plasma for different time: (a) untreated PEDOT:PSS, (b) 3 min.

confirmed by the cross-section SEM images as shown in Fig. 3 . The thickness of untreated and 3 min treated films are $31.3 \mathrm{~nm}$ and $29.2 \mathrm{~nm}$ respectively, which is in accordance with the Profilometer measurements. The above results indicated that the plasma treatment process could remove materials from the surface of PEDOT:PSS layer quite slightly, which was slower than the removal rate of approximately $5 \mathrm{~nm} \mathrm{~min}{ }^{-1}$ presented in the literature. ${ }^{11}$

The surface morphology of PEDOT:PSS films was also examined by AFM as shown in Fig. 4, which confirmed the observation from SEM. Initially, AFM image showed nanometer-scale islands with a size of approximately $100 \mathrm{~nm}$ (Fig. 4a) on the untreated PEDOT:PSS layer, suggesting a rootmean-square (RMS) of $1.12 \mathrm{~nm}$. After 1 min treatment, small particles were found on the surface, which is ascribed to the removal of featureless top PSS chains from the surface ${ }^{15,16}$ (Fig. $4 \mathrm{~b})(\mathrm{RMS}=1.14 \mathrm{~nm})$. With increased treatment time, the density of particles increased and the edge of the particles became shaper (Fig. 4c), and PEDOT:PSS surface showed more uniformed and more condensed grains. After 5 min treatment, the particles gradually connected to the adjacent ones and grown up to islands around $20 \mathrm{~nm}(\mathrm{RMS}=1.26 \mathrm{~nm})$. It should be noticed that much rougher surface was found for argon plasma treated PEDOT:PSS films (Fig. 4b-d), among which
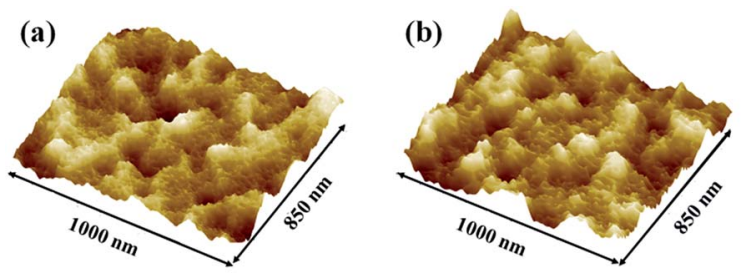

(c)
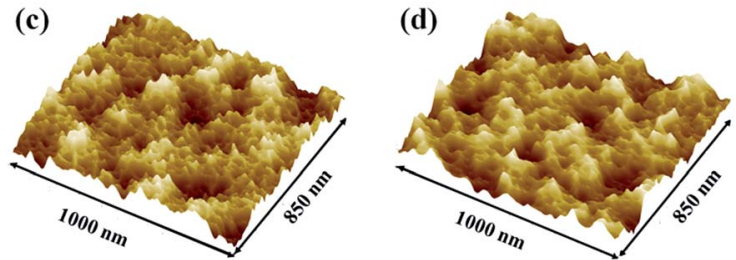

Fig. 4 AFM surface images of PEDOT:PSS treated by argon plasma for different time: (a) untreated, (b) $1 \mathrm{~min}$, (c) $3 \mathrm{~min}$, (d) $5 \mathrm{~min}$.
3 min treated PEDOT:PSS film formed roughest surface (RMS = $1.36 \mathrm{~nm})$.

To investigate the possible changes in the film conductivity, the conductivity of PEDOT:PSS film $(\sigma)$ was calculated according to the following formulation: $\sigma=\frac{1}{R_{\mathrm{S}} \times d} . R_{\mathrm{S}}$ and $d$ represent the sheet resistance and the thickness of measured film, respectively. $R_{\mathrm{S}}$ was measured by a four-point probe method with a Keithley Instrument Model B2902A. To minimize the effect of the contact resistance between the PEDOT:PSS film and the probe, silver-indium layer was thermally deposited on the PEDOT:PSS surface as top electrode.

The conductivity is $12.8 \mathrm{mS} \mathrm{cm}^{-1}$ for pristine PEDOT:PSS film, and $14.3 \mathrm{mS} \mathrm{cm}^{-1}, 15.5 \mathrm{mS} \mathrm{cm}^{-1}, 17.9 \mathrm{mS} \mathrm{cm}^{-1}$ for the films treated by argon plasma for $1 \mathrm{~min}, 3 \mathrm{~min}$ and $5 \mathrm{~min}$, respectively. The plasma-treated PEDOT:PSS layers exhibited enhanced conductivity, which may be attributed to the enlarged ratio of PEDOT to PSS. Normally, the PEDOT-to-PSS molar concentration ratio in films strongly affects the electrical conductivity and sheet resistance. The higher PEDOT concentration, the larger the electrical conductivity corresponding charge transporting layer. ${ }^{17}$

XPS spectra was measured to determine the surface component of PEDOT:PSS films modified by argon plasma posttreatment. Fig. 5 shows the S 2p spectra of pristine PEDOT:PSS films and treated films. Two binding energy components are observed at 164.0 and $168.2 \mathrm{eV}$, which are corresponding to PEDOT and PSS respectively. ${ }^{18,19}$ The $\mathrm{S} 2 \mathrm{p}$ contributing peaks at 163.9 and $165.3 \mathrm{eV}$ result from the spin-split doublets of the sulfur atoms in PEDOT. On the other hand, the S signals from PSS are present at 166.5-171.0 eV because high-electronegative oxygen atoms are attached to sulfur fragments of PSS. ${ }^{20}$ The higher binding energy component of the $\mathrm{S} 2 \mathrm{p}$ peak at $169.2 \mathrm{eV}$ (green line, Fig. 5) is associated with the $\mathrm{SO}_{3}-\mathrm{H}$ group of PSS, whereas the lower binding energy component of the $\mathrm{S} 2 \mathrm{p}$ peak at $168.1 \mathrm{eV}$ (pink line, Fig. 5) corresponds to the $\mathrm{SO}_{3}{ }^{-}$group of

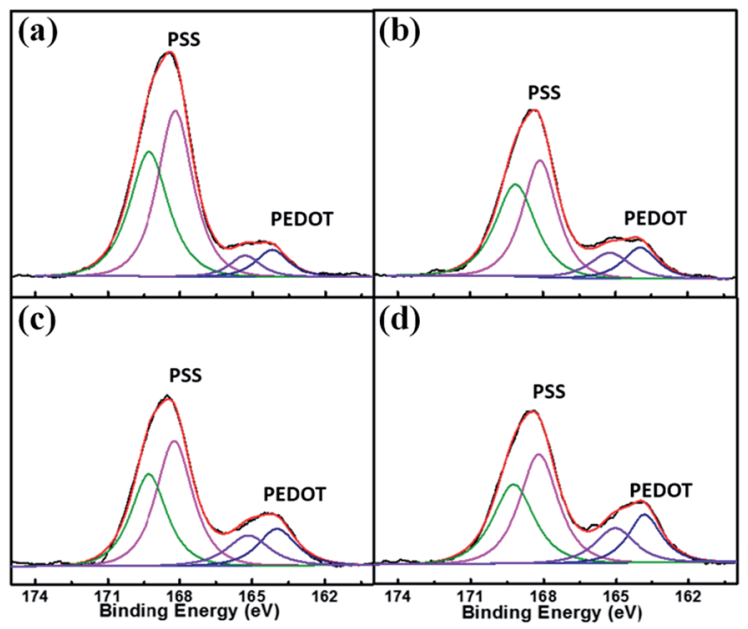

Fig. $5 \mathrm{~S}(2 \mathrm{p})$ core level spectrum for PEDOT:PSS films treated by argon plasma for different time: (a) untreated, (b) $1 \mathrm{~min}$, (c) $3 \mathrm{~min}$, (d) 5 min. 
PSS. ${ }^{21}$ With the increase of treatment time, the peak areas rate of PEDOT to PSS increased from 0.14 (untreated) to $0.27,0.33$, and 0.40 , respectively, indicating that PEDOT-to-PSS molar concentration ratio are significantly enhanced during low energy argon plasma treatment, which is in good accordance with the aforementioned conductivity results. Unfortunately, the mechanism of the increasing PEDOT-to-PSS ratio by mild argon plasma treatment has not been fully understood yet. As descripted in the ref. 22 and 23, there is a layer of excess PSS with the thickness of approximately $32-43 \AA$ surrounding the PEDOT-PSS grains at the surface. During argon plasma treatment, the excess PSS is easily removed away from the surface of the films, which is likely ascribed to the change of chemical compositions.

The interfacial property of hole transport layers plays a critical role on the deposition of perovskite layers. To further study the surface wettability of PEDOT:PSS films, the contact angle measurements between the droplet of perovskite precursor solution and PEDOT:PSS films were executed at the same time. The difference between the argon plasma treated PEDOT:PSS films and un-treated PEDOT:PSS is compared. It is found that the contact angel of perovskite precursor solution on the untreated PEDOT:PSS films is $11.2^{\circ}$. After different argon plasma treatment time, the angle decreases to $8.9^{\circ}, 7.2^{\circ}, 7.1^{\circ}$, respectively.

It is suggested that the wettability can be significantly improved by argon plasma post-treatment, which can be mainly explained by the increased surface free energy. ${ }^{24}$ It also could be partially explained by the condensed particles or islands appeared on the treated surface which leads to a relatively higher specific surface area. Another reason is that the contamination or dust particle on surface of PEDOT:PSS may be removed by the plasma cleaning process. Fig. 6 shows images of final morphology by dropping $2.5 \mu \mathrm{L}$ perovskite precursor

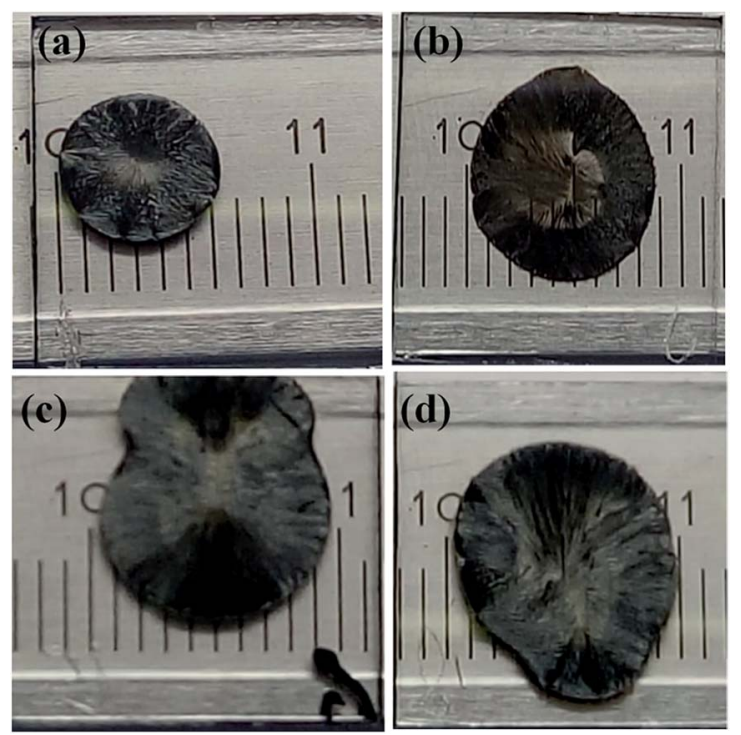

Fig. 6 The morphology of perovskite precursor solution dropped on the PEDOT:PSS films for different time: (a) untreated PEDOT:PSS, (b) $1 \mathrm{~min}$, (c) $3 \mathrm{~min}$, (d) $5 \mathrm{~min}$. solution on the PEDOT:PSS films after argon plasma treatment for $0 \mathrm{~min}, 1 \mathrm{~min}, 3 \mathrm{~min}, 5 \mathrm{~min}$, respectively. It was seen that perovskite precursor solution is dispersed more easily on the argon plasma treated PEDOT:PSS films. Meantime, the surface wettability of treated PEDOT:PSS films and perovskite precursor solution is also found to be improved because of the bigger solution area in the pictures.

\subsection{The performance of the devices}

Inverted planar heterojunction perovskite solar cells with the structure of ITO/PEDOT:PSS/ $\mathrm{CH}_{3} \mathrm{NH}_{3} \mathrm{PbI}_{3} / \mathrm{PC}_{61} \mathrm{BM} / \mathrm{Ag}$ were fabricated to study the influence of Ar plasma post-treatment on the cells' performance. The morphology and crystalline of the perovskite films on different treated PEDOT:PSS were characterized, as shown in Fig. S1. $\dagger$ It is found that the uniform grains could be obtained on the treated PEDOT:PSS, and the best morphology was grown on 3 min-treated one. The $J-V$ characteristics and photovoltaic performance data corresponding to the power conversion efficiency (PCE) for various treating time were shown in Fig. 7.

The pristine PEDOT:PSS films based device gave an open circuit voltage $\left(V_{\mathrm{oc}}\right)$ of $0.86 \mathrm{~V}$, a short circuit current density $\left(J_{\mathrm{sc}}\right)$ of $16.72 \mathrm{~mA} \mathrm{~cm}^{-2}$, a fill factor (FF) of 0.66 and a PCE of $9.65 \%$. As the treatment time increased, the $V_{\text {oc }}$ increased to $0.87 \mathrm{~V}$, $0.88 \mathrm{~V}, 0.92 \mathrm{~V}$ for $1 \mathrm{~min}, 2 \mathrm{~min}$ and $3 \mathrm{~min}$, respectively. It reached a maximum of $0.94 \mathrm{~V}$ at $4 \mathrm{~min}$, and then dropped down to $0.87 \mathrm{~V}$ at $5 \mathrm{~min}$. Compared with the untreated PEDOT:PSS film based device, the $J_{\mathrm{sc}}$ increased from $16.72 \mathrm{~mA} \mathrm{~cm}^{-2}$ to 19.14 $\mathrm{mA} \mathrm{cm}{ }^{-2}$ excitingly, corresponding to the $3 \mathrm{~min}$ treatment. On the contrary, the argon plasma treatment has less influence on the value of FF except for 3 min treatment. Compared with the untreated PEDOT:PSS film based device, the PCE increased from $9.55 \%$ to $9.62 \%, 10.78 \%, 12.17 \%$. Therefore, it indicates that an appropriate treating with argon plasma can effectively improve the performance of PSCs, which can be attributed to the enhanced $V_{\mathrm{oc}}$ and $J_{\mathrm{sc}}$.

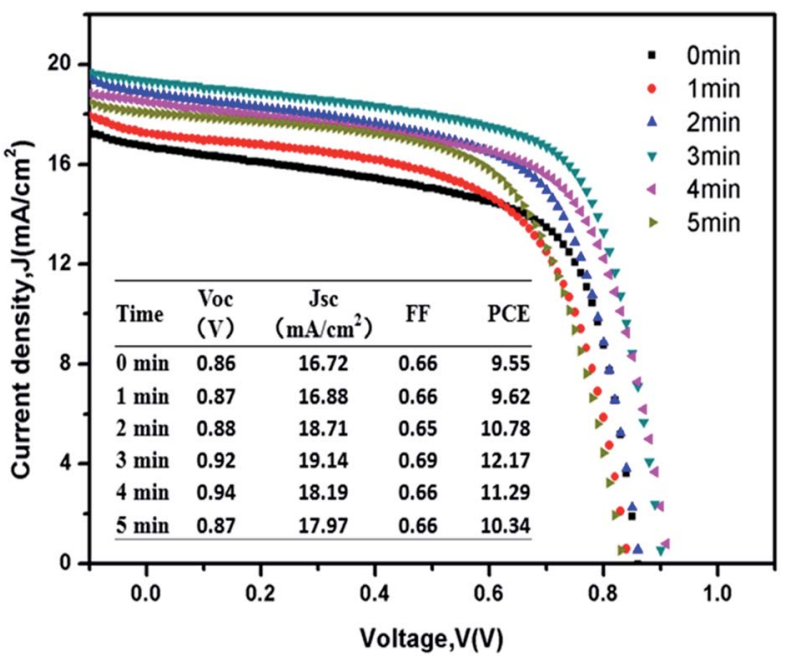

Fig. 7 J-V curves and PV performance data of inverted planar PSCs based on PEDOT:PSS films treated by argon plasma as a function of treatment time. 
The $J_{\text {sc }}$ is found to be improved markedly by argon plasma post-treating on the surface of PEDOT:PSS films, which is attributed to an enhanced charge collection efficiency resulting from improved wettability between the perovskite layer and PEDOT:PSS layer. ${ }^{8}$ A higher conductivity caused by the removal of PSS concentration from XPS analysis may contribute to the improved $J_{\text {sc }}$. The significantly increased value of $V_{\text {oc }}$ could be explained by the changing of HOMO and LUMO band gap level presented by Attri, et al., ${ }^{25}$ which leads to a restrained charge recombination. It should be noticed that, the devices based on PEDOT:PSS layer treated by $4 \mathrm{~min}$ and $5 \mathrm{~min}$ exhibit reduced efficiency of $11.29 \%$ and $10.34 \%$, which are still higher than the efficiency of devices based on pristine PEDOT:PSS layer. In order to study the repeatability, 5 series of experiments at the same conditions have been executed, and the results showed that argon plasma post-treatment on PEDOT:PSS could effectively enhance the performance of inverted planar heterojunction perovskite solar cells, even though a certain fluctuation is presented, as shown in Fig. S2. $\dagger$

\section{Conclusions}

In summary, we have systematically studied the influence of mild argon plasma post-treatment on the properties of the PEDOT:PSS films, the subsequent interface wettability between perovskite and PEDOT:PSS films, as well as the PSCs performance. XPS analysis confirmed that PSS component was partially removed, leading to an enhanced conductivity of a PEDOT-rich surface. SEM and AFM analysis revealed that argon plasma treated PEDOT:PSS surface appeared uniformly distributed islands with increasing surface roughness and consequently led to better wettability interface between perovskite film and PEDOT:PSS films. Although the PEDOT to PSS molar concentration ratio and surface morphology were modified, and the layer thickness was found to decrease slightly $\left(0.8 \mathrm{~nm} \mathrm{~min}^{-1}\right)$ for those plasma-treated PEDOT:PSS layers, which was evidenced by cross sectional SEM images and profilometer measurements. PSCs based on argon plasma treated PEDOT:PSS films were fabricated, and corresponding efficiency of PSCs remarkably increased from $9.55 \%$ for the pristine PEDOT:PSS films based device to $12.17 \%$ for the 3 min-treated PEDOT:PSS film. We believe that this method may be beneficial for enhancing the efficiency of all PSCs.

\section{Acknowledgements}

This work was financially supported by National Key Basic Research Program of China (2014CB931702), NSFC (91421110 and 61604032), Sichuan Provincial Fund for Distinguished Young Academic and Technology Leaders (2014JQ0011). M. Y., X. H. and S. W. contributed equally to this work.

\section{References}

1 J. J. Nam, J. Noh, W. S. Yang, Y. C. Kim, S. Ryu, J. Seo and S. I. Seok, Nature, 2015, 517, 476-480.
2 X. Li, D. Bi, C. Yi, J. Decoppet, J. Lou, S. M. Zakeeruddin, A. Hagfeldt and M. Grätzel, Science, 2016, 353, 58-62.

3 D. P. McMeekin, G. Sadoughi, W. Rehman, G. E. Eperon, M. Saliba, M. T. Hörantner, A. Haghighirad, N. Sakai, L. Korte, B. Rech, M. B. Johnston, L. M. Herz and H. J. Snaith, Science, 2016, 351, 151-155.

4 M. A. Green, A. Ho-Baillie and H. J. Snaith, Nat. Photonics, 2014, 8, 506-514.

5 A. Kojima, K. Teshima, Y. Shirai and T. Miyasaka, J. Am. Chem. Soc., 2009, 131, 6050-6051.

6 http://www.nrel.gov/ncpv/images/efficiency_chart.jpg.

7 L. Jiang, S. Cong, Y. Lou, Q. Yi, J. Zhu, H. Ma and G. Zou, J. Mater. Chem. A, 2016, 4, 217.

8 W. Li, H. Dong, X. Guo, N. Li, J. Li, G. Niu and L. Wang, J. Mater. Chem. A, 2014, 2, 20105-20111.

9 X. Li, L. Zhang, F. Tang, Z. Bao, J. Lin, Y. Li, L. Chen and C. Ma, RSC Adv., 2016, 6, 24501-24507.

10 Z. Su, L. Wang, Y. Li, H. Zhao, B. Chu and W. Li, Nanoscale Res. Lett., 2012, 7, 465.

11 Y. Zhou, Y. Yuan, J. Lian, J. Zhang, H. Pang, L. Cao and X. Zhou, Chem. Phys. Lett., 2006, 427, 394-398.

12 T. Ino, T. Hayashi, K. Ueno and H. Shirai, Thin Solid Films, 2011, 519, 6834-6839.

13 T. Ino, T. Hayashi, T. Fukuda, K. Ueno and H. Shirai, J. Nanosci. Nanotechnol., 2011, 11, 8035-8039.

14 M. Xiao, F. Huang, W. Huang, Y. Dkhissi, Y. Zhu, J. Etheridge, A. Gray-Weale, U. Bach and Y. Cheng, Angew. Chem., Int. Ed., 2014, 53, 9898-9903.

15 J. Ouyang, Q. Xu, C. W. Chu, Y. Yang, G. Li and J. Shinar, Polymer, 2004, 45, 8443-8450.

16 J. S. Yeo, J. M. Yun, D. Y. Kim, S. Park, S. S. Kim, M. H. Yoon, T. W. Kim and S. I. Na, ACS Appl. Mater. Interfaces, 2012, 4, 2551-2560.

17 I. Tomohisa, H. Tatsuya, U. Keiji and S. Hajime, Thin Solid Films, 2011, 519, 6834-6839.

18 S. A. Spanninga, D. C. Martin and Z. Chen, J. Phys. Chem. C, 2010, 114, 14992-14997.

19 J. Wang, R. S. Karmakar, Y. Lu, M. Wu and K. Wei, J. Phys. Chem. C, 2016, 120, 25977-25984.

20 X. Crispin, S. Marciniak, W. Osikowicz, G. Zotti, A. W. Denier van der Gon, F. Louwet, M. Fahlman, L. Groenendaal, F. De Schryver and W. R. Salaneck, J. Polym. Sci., Part B: Polym. Phys., 2003, 41, 2561-2583.

21 G. Greczynski, T. Kugler and W. R. Salaneck, Thin Solid Films, 1999, 354, 129-135.

22 S. K. M. Jönsson, J. Birgerson, X. Crispin, G. Greczynski, W. Osikowicz, A. W. Denier van der Gon, W. R. Salaneck and M. Fahlman, Synth. Met., 2003, 139, 1-10.

23 G. Greczynski, T. Kugler, M. Keil, W. Osikowicz, M. Fahlman and W. R. Salaneck, J. Electron Spectrosc. Relat. Phenom., 2001, 121, 1-17.

24 B. D. Beake, J. S. G. Ling and G. J. Leggett, J. Mater. Chem., 1998, 8, 2845-2854.

25 P. Attri, V. Bharti, Y. S. Kim, J. Gaur, S. Chand, G. Kwon, S. Lee, W. Lee, E. H. Choi and I. T. Kim, Phys. Chem. Chem. Phys., 2014, 16, 27043-27052. 